EP-26

\title{
Herbal and traditional therapies for liver disease: A study with rural communities in Indonesia
}

\author{
Nuraliah NURALIAH* $^{*}$
}

Public Health, West Sulawesi Research and Empowerment Center, Indonesia

Introduction: Liver disease is one of the leading causes of death in Indonesia. In 2013, it was reported that Indonesia's hepatities prevalence was $1.2 \%$. This study aims to describe people's experience in preventing liver disease. The authors explore the medical plants that was used by the patient to cure liver by using herbal and therapies.

Methods: This study used literature review. The author collected data from some journals to study the local knowledge of rural communities to overcome liver disease.

Results: The phenomenon learned from this treatment was not only the behavior of people who use alternative medicine to prevent various disease, but also the effectiveness of these traditional therapies. The results of this study indicate that: 1) herbs have become the main treatment choice for rural communities; 2) therapies are chosen because it has been a treatment for generetions.

Conclusions: Base on the results of the study it can be concluded that the majority of rural people in Indonesia develop more and use herbs as alternative treatments to overcome liver. Local therapy was the main outcome measure and it had the role in reducing various disease. 